\section{Farbstoff-Anaphylaxie!}

$\mathrm{M}$ it Etablierung der Wächterlymphknotenbiopsie bei Karzinomoperationen muss jetzt vermehrt auch an anaphylaktische Reaktionen gegen die zur Lymphknotenlokalisation perioperativ injizierten Blaufarbstoffe gedacht werden. Diese Anaphylaxien verlaufen oft schwer und treten typischerweise mit einer Verzögerung von etwa 30 Minuten nach der Injektion auf. Die Symptome können über mehrere Stunden persistieren und erfordern dann eine kontinuierliche Adrenalininfusion, so das Ergebnis einer Fallserie aus Frankreich mit 14 Brustkrebspatientinnen, die nach der intraoperativen Injektion von Patentblau (E 131) anaphylaktische Reaktionen entwickelt hatten. Die Sensibilisierungen lassen sich

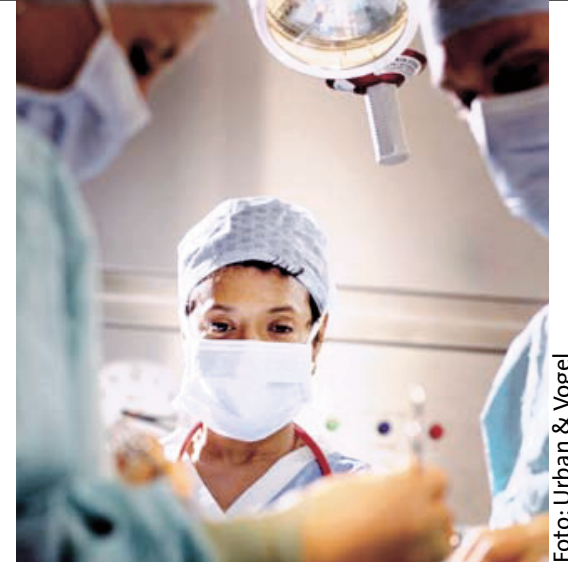

meist mit Hauttests nachweisen, bei negativem Pricktestergebnis sollte zusätzlich intradermal getestet werden. Bei positiver Reaktion gegen Patentblau oder andere höhermolekulare Blaufarbstoffe bietet sich Methylenblau als Alternative an. $\quad b k$

Mertes PM et al. J Allergy Clin Immunol 2008; 122: 348-52

\title{
Schwimmen tut Asthmakranken gut
}

chwimmsportler haben ein erhöhtes Asthmarisiko. Unklar war bisher, ob es dabei einen kausalen Zusammenhang mit dem Aufenthalt in chlorhaltigem Schwimmbadwasser gibt. Diese Frage sollte jetzt eine US-amerikanische Metaanalyse klären. Die Untersuchung bestätigte die erhöhte Asthmainzidenz bei TopSchwimmern im Vergleich zu anderen Spitzenathleten. Inkonsistent war dagegen das Ergebnis zu Asthma und Hallenbadbesuchen in der Kindheit. Weitere Berech- nungen ergaben, dass Asthmakranke eher von einem Schwimmtraining profitieren und dass Schwimmen weniger stark den Atemwegsswiderstand erhöht als andere Sportarten. Vielleicht haben sich also Asthmapatienten Schwimmen als ,ihre“ Sportart ausgesucht. Chlorexposition als Wegbereiter für Asthma wird in dieser Metaanalyse dagegen eher ausgeschlossen. $b k$

Goodman M et al. J Asthma 2008; 45:

639-47

\section{Staubgetragene Staphylokokken-Kolonisation}

B ei rund $90 \%$ aller Patienten mit einer atopischen Dermatitis liegt eine Kolonisation der Haut mit Staphylococcus aureus vor. Superantigene und Zellwandbestandteile der grampositiven Bakterien verschlechtern das Krankheitsbild, weshalb die bakterielle Belastung möglichst niedrig gehalten werden muss. Die entsprechenden Präventionsempfehlungen müssen jetzt wohl

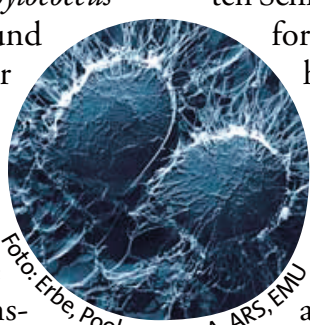

nämlich, dass die S.-aureus-Konzentrationen im Staub der Betten und des gesamten Schlafzimmers bei schweren Verlaufsformen der Erkrankung besonders hoch sind. Die Konzentrationen korrelierten direkt mit der Schwere des Ekzems. Möglicherweise ist der Bett- und Schlafzimmerstaub eine wichtige Reexpositionsquelle, die den Erfolg anderer therapeutischer Maßnahmen schmälert.

$b k$ um eine weitere Maßnahme ergänzt werden: Wohnraumuntersuchungen bei Patienten mit atopischer Dermatitis ergaben
Leung AD et al. Clin Exp Allergy 2008; 38 : 789-93

\section{Späte Einsicht wirkt}

- $s$ gleicht manchmal einem Kampf - gegen Windmühlenflügel, einen Raucher zum Nichtrauchen zu bewegen. An der Einsicht des Betroffenen mangelt es selten, an der Umsetzung häufig. Der beste Zeitpunkt für eine ärztliche Gardinenpredigt in puncto Lebensstil ist wohl gekommen, wenn eine dadurch verursachte Erkrankung neu diagnostiziert wird. Die Chance, dass ein Patient dann mit dem Rauchen aufhört, liegt - je nach Diagnose - um den Faktor 2,9 bis 6,1 höher. de

Keenan PS. Arch Intern Med 2009; 169:

237-42

\section{Marathon und Melanom}

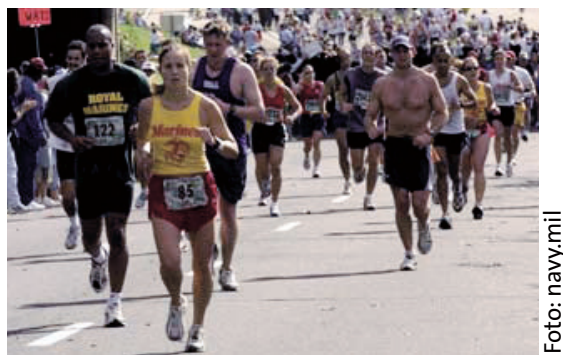

M arathonläufer haben ein erhöhtes Melanomrisiko. Dies zeigt erneut eine Studie, in der bei 150 aktiven Marathonläufern eine erhöhte Zahl von Lentigines und Naevi auf der linken Schulter im intraindividuellen Vergleich zum Gesäß nachgewiesen wurde. Die Anzahl der durchgemachten Sonnenbrände und die Art der Sportbekleidung korrelierte positiv mit der Zahl der Lentigines, die Trainingsintensität bzw. körperliche Belastung im Training beeinflusste die Zahl der Naevi. Eine erhöhte Anzahl von Naevi und Lentigines an sonnenexponierten Körperregionen ist von anderen Outdoor-Sportlern bekannt. Durch funktionelle Sportkleidung, wasserfestes Sonnenschutzmittel sowie die Verlegung der Trainingszeiten in die Morgen- und Abendstunden wird die UV-Belastung vermindert. Gleichzeitig sollten ein „Übertraining" und eine damit möglicherweise verbundene Immunsuppression vermieden werden. $b k$

Richtig E et al. Dermatology 2008; 217: 3844 